\title{
A spatial model of political competition and proportional representation
}

\author{
Ignacio Ortuño-Ortín
}

University of Alicante and Instituto Valenciano de Investigaciones Economicas

\begin{abstract}
A spatial model of party competition is studied in which: (i) Parties are supposed to have ideology. By this we mean that their goal is to maximize the welfare of their constituencies. (ii) The policy implemented after the election does not need to coincide with the one proposed by the winner. The policy implemented should be a compromise that considers the proposals made by the different parties. In the case of proportional representation this compromise is modeled as a convex combination of the proposed policies with weights proportional to the number of votes obtained by each party. We provide some existence theorems and compare the equilibrium in our model with the equilibrium that exists under some probabilistic models. It is also shown that proportional representation will create incentives for the parties to announce radical platforms.
\end{abstract}

\section{Introduction}

In the classical Hotelling-Downs model of elections (Hotelling 1929; Downs 1957) it is assumed that there exists a one-dimensional and compact space in which the policies proposed by parties can be seeing as points. Agents have well-defined preferences over such space and vote for the party whose proposal is closest to their most preferred point. The objective function of parties is to win the election. To achieve this goal, each party proposes the policy that maximizes the votes in its favor, taking the other party's proposal as fixed.

The author is grateful to Luis Corchón, John Roemer and Christian Shultz for many helpful comments, as well as to seminar participants in Universities of Copenhagen and Alicante. I also thank an anonymous referee for helpful suggestions. Financial support from Spanish Ministry of Education, projects no. PB93-0940 and PB94-1504 and a D.G.I.C.Y.T. mobility grant is gratefully acknowledged. 
This approach has produced very important insights and conclusions. The most famous of these conclusions is the "principle of minimum differentiation". In the two party case, under certain strong assumptions, the policies proposed converge toward the median voter's most preferred point. During the last two decades many extensions and modifications of the basic model have been proposed. Because of these modifications "minimum differentiation" is not longer accepted as a general principle. The basic insight, however, is still valid: parties have strong incentives to converge in their proposed policies (see an excellent edited survey on spatial political competition by Enelow and Hinich (1990), and a more recent one on political economy by Barnett et al. (1993)). However if the policy space is of high dimension then an equilibrium may not exist.

Most of these extensions and modifications share a basic assumption of the original Hotelling-Downs model: the objective function of the parties. They continue characterizing a political party as an institution whose goal is to win the election. Some authors think, however, that it is not clear that the objective function of parties is best modeled by maximization of vote support. Ginsburgh et al. (1987) Wittman (1983, 1990), Calvert (1985), Roemer (1993, 1994) and Shultz (1995) assume that parties are "ideological", meaning that they have preferences over the space of admissible policies. Their objective here will be to adopt the strategy that will induce the best possible policy according to their preferences. Those authors also assume that there is uncertainty about the outcome of the election. The result now is that, in general, parties' proposals do not converge.

In this paper it is also assumed that parties are ideological . Unlike the authors just mentioned we do not introduce uncertainty in the model. Our distinctive feature will be related to the outcome of the political game. All the formal models in the literature suppose that the winning party has the power to implement its proposed policy (at least in the two-party case). We do not accept this as a realistic and satisfactory way to model the outcome of the political competition in many modern democratic societies. A democratic society is formed by different institutions and groups that are relevant to the political decisions: parliament, trade unions, media, lobbies and so on. Some of them may be more in favor of the policy that was announced by the defeated party; others, on the contrary, may be in favor of the winning party's proposal. All of them may have some influence on the actions taken by the government (who is, perhaps, formed by the winning party). The influence of groups in favor of a proposal will be a nondecreasing function of the proportion of votes obtained by the corresponding party. Clearly a party winning $51 \%$ of the votes will have more difficulty to carry out its proposal than one winning $80 \%$ of the votes. In general this influence will not be proportional to the vote share (even more, this relation may be discontinuous, "going from $(50-\varepsilon) \%$ to $(50+\varepsilon) \%$ of the vote, no matter how small is $\varepsilon$, can be more valuable than going from $47 \%$ to $49 \%$ ", Denzau et al. 1985, p. 96).

This view motivates our assumption that, in the two-party system, the implemented policy will be a convex combination of the parties' proposals. The weights that determine this convex combination will be given by a nondecreasing function in the vote share. Clearly, the specific function used should depend on many institutional and cultural factors. 
We confine ourselves to the analysis of what we call the Proportional Representation case. Under Proportional Representation the weight associated to a proposal is proportional to the vote share. One might think that in actual democracies implemented policies are not proportional convex combinations of parties proposals and, consequently, our approach is an unrealistic one. But the standard Hotelling-Downs approach can be criticized also as very unrealistic: it is hard to believe that it makes no difference to win with $51 \%$ or to win with $90 \%$ of the votes. Thus, our view is that despite the unrealism of the Proportional Representation model it is worth studying for much the same reasons that the standard Hotelling-Downs model is worth studying. Both models are polar cases that should be understood before analyzing more realistic ones.

The results obtained in this paper show that under Proportional Representation, for the two-party system, parties' proposals do not converge toward centrist platforms. Furthermore, there is an incentive to become "radical", i.e., to put forth proposals that are more extreme than the parties "ideal" policies (see Poole and Rosenthal (1984, 1991) for empirical evidence of a large divergence in political platforms). The intuition behind this result is clear. Consider for example the "Left" party. A move toward more extreme left positions produces two effects. On the one hand, the number of votes in favor of this party decreases. Thus, the weight associated to its policy will also decrease. On the other hand, this weight is now put on a more leftist policy. The net effect can go either way and it will depend on the distribution of voters. We characterize cases for which the second effect overcomes the first one.

The organization of the rest of the paper is as follows. Section 2 sets up the basic model. Some results on the existence of equilibrium are presented in Sect. 3. Section 4 provides a more specific model in which agents have symmetric utility functions and compares the results with the ones obtained under some probabilistic models. Section 5 concludes and proposes some issues for further research.

\section{The model}

Let $S=\left[s_{m}, s_{M}\right] \subset R$ be the set of agents' types and $T=\left[t_{m}, t_{M}\right] \subseteq R_{+}$ the set of admissible policies. Agent of type $s \in S$ has utility function $v(. ; s): T \rightarrow R$. The distribution of types is given by an (absolutely continuous) probability measure $F$ on $S$. Let $t(s) \in T$ be a most preferred policy by an agent of type $s$, i.e., $t(s)$ is such that $v(t(s) ; s) \geq v(t ; s)$ for all $t \in T$. By part (a) in the following assumption $t(s)$ always exists and is unique.

Assumption 1. (a) $v(t ; \mathrm{s})$ is strictly concave and differentiable in $t$. (b) $v(t ; s)$ is continuous in $s$. (c) $t(s)$ is strictly increasing in $s$.

There are two parties denoted by $L$ and $R$. Party $L$ has a utility function over the set of admissible policies $\Pi_{L}: T \rightarrow R$ that coincides with the utility function of agent of type $s_{L}$, i.e., $\Pi_{L}(t)=v\left(t ; s_{L}\right)$ for all $t$. In a similar way party $R$ has utility function $\Pi_{R}: T \rightarrow R$ such that $\Pi_{R}(t)=v\left(t ; s_{R}\right)$. Consequently the "ideal" policy for party $L$ is $t\left(s_{L}\right)=l$ and the ideal one for party $R$ is $t\left(s_{R}\right)=r$. Thus we think of the constituencies of party $L$ and $R$ as formed by agents of 
type $s_{R}$ and $s_{L}$ respectively ${ }^{1}$. We will suppose that $\mathrm{s}_{L}<s_{M V}<s_{R}$ where $s_{M V}$ is the median type, i.e. $F\left(\left(s: s \leq s_{M V}\right)\right)=1 / 2$.

The political game is such that each party announces a policy which must be an element of $T$. Each agent votes for the party that announced the policy which gives a higher utility according to his utility function. Let $t_{L} \in T$ be the policy announced by party $L$ and $t_{R} \in T$ the one announced by party $R$. Given this pair of proposals, $\left(t_{L}, t_{R}\right)$, type s agent votes with probability one for party $L(R)$ iff $v\left(t_{L} ; s\right)>v\left(t_{R} ; \mathrm{s}\right)\left(\right.$ iff $\left.v\left(t_{L} ; s\right)<v\left(t_{R} ; s\right)\right)$ and whenever $v\left(t_{L} ; s\right)=v\left(t_{R} ; s\right)$ he votes for party $L(R)$ with probability $1 / 2$. Note that the set of agents that would be indifferent between two policies will have measure zero. Let $\Omega\left(t_{L}, t_{R}\right)$ be the set of agents who strictly prefer $t_{L}$ rather than $t_{R}$, i.e., $\Omega\left(t_{L}, t_{R}\right) \equiv\{s \in S$ : $\left.v\left(t_{L} ; s\right)>v\left(t_{R} ; s\right)\right\}$ and $E\left(t_{L}, t_{R}\right)$ the set of indifferent agents, $E\left(t_{L}, t_{R}\right) \equiv\{s \in S$ : $\left.v\left(t_{L} ; s\right)=v\left(t_{R} ; s\right)\right\}$. Party $L$ obtains the proportion of votes given by $\Psi\left(t_{L}, t_{R}\right)=F\left(\Omega\left(t_{L}, t_{R}\right)\right)+1 / 2 F\left(E\left(t_{L}, t_{R}\right)\right)$. The functions $v(t ; s)$ and $F$ are supposed to be common knowledge.

Now we departure from most theoretical models on political competition: it is assumed that the implemented policy after the election takes place doesn't need to be the one announced by the winner. We suppose that the implemented policy, which we denote by $t\left(t_{L}, t_{R}\right)$, is a convex combination of the parties proposals with weights that depend on the percentage of votes obtained by each party:

$$
t\left(t_{L}, t_{R}\right)=W\left(\Psi\left(t_{L}, t_{R}\right)\right) t_{L}+\left(1-W\left(\Psi\left(t_{L}, t_{R}\right)\right)\right) t_{R},
$$

where $W:[0,1] \rightarrow[0,1]$.

Several cases can be studied:

The Standard Model: Here the party that gets more than $50 \%$ of the votes has the power to carry out its announced policy. Thus the winner takes all and $W$ is given by

$$
W_{s}\left(\Psi\left(t_{L}, t_{R}\right)= \begin{cases}0 & \text { if } \Psi\left(t_{L}, t_{R}\right)<1 / 2 \\ 1 / 2 & \text { if } \Psi\left(t_{L}, t_{R}\right)=1 / 2 \\ 1 & \text { if } \Psi\left(t_{L}, t_{R}\right)>1 / 2\end{cases}\right.
$$

Proportional Representation: Here the "influence" of the groups in favor of a given policy is proportional to the percentage of votes in favor of that policy. The weights are now given by

$$
W_{P R}\left(\Psi\left(t_{L}, t_{R}\right)\right)=\Psi\left(t_{L}, t_{R}\right) .
$$

Most of the literature modeling party competition adopts the Standard Model. In this paper, on the contrary, only the Proportional Representation model will be analyzed. We believe that a more realistic approach should consider a weight function $W$ that is between these two extreme cases.

Thus in the rest of the paper we assume that, after the election takes place, the policy implemented is $t\left(t_{L}, t_{\mathrm{R}}\right)=\Psi\left(t_{L}, t_{\mathrm{R}}\right) t_{L}+\left(1-\Psi\left(t_{L}, t_{\mathrm{R}}\right)\right) t_{R}$. The utility obtained by each party depends on this implemented policy $t\left(t_{L}, t_{R}\right)$. Then,

\footnotetext{
${ }^{1}$ Nothing essential would change if we allow for constituencies with more than just one type of agents.
} 
the objective of a party $L$ is to choose the policy $t_{L}$ that solves the following maximization problem (and similarly for party $\mathrm{R}$ ):

$$
\max _{t_{L}} \Pi_{L}\left(t\left(t_{L}, t_{R}\right)\right) .
$$

Definition. A Political Equilibrium is a vector of policies $t^{*}=\left(t_{L}^{*}, t_{R}^{*}\right) \in T \times T$ such that

(a) $\Pi_{L}\left(t\left(t_{L}^{*}, t_{R}^{*}\right)\right) \geq \Pi_{L}\left(t\left(t, t_{R}^{*}\right)\right)$ for all $t \in T$,

(b) $\Pi_{R}\left(t\left(t_{L}^{*}, t_{R}^{*}\right)\right) \geq \Pi_{R}\left(t\left(t_{L}^{*}, t\right)\right)$ for all $t \in T$.

Observe that in our model only parties behave strategically. They care about the implemented policy and choose their strategies (proposals) to influence toward their desired directions. Voters, however, always vote "sincerely". An agent votes for the policy that, were it implemented, would give him a higher utility level. So voters only compare the two proposals and no the possible final outcomes. Thus, agents are assumed to be less sophisticated than parties. This is a common restriction in this area of research ${ }^{2}$.

To obtain positive results we need to introduce some further restrictions on the admissible preferences.

Assumption 2. (Single Crossing Property). For any policies $t^{\prime}, t^{\prime \prime} \in T, t^{\prime} \neq t^{\prime \prime}$, there is at most one type $s \in S$ such that $v\left(t^{\prime} ; s\right)=v\left(t^{\prime \prime} ; s\right)$.

Assumption 3. $t\left(s_{m}\right)=t_{m}$ and $t\left(s_{M}\right)=t_{M}$.

The Single Crossing Property assumption is standard in economics and it is needed to have a "nice" separation of the set of agents for and the set of agents against a given policy. Assumption 3 is basically technical and it is not necessary if we assume that for some $s \in S$ we have $t(s) \in\left(t_{m}, t_{M}\right)$.

Lemma 1. Under assumptions $1-3$, and for any $\left(t_{L}, t_{R}\right) \in T \times T, t_{L}<t_{R}$, there exists a type $s_{d}\left(t_{L}, t_{R}\right) \in S$ such that all agents with type $s<s_{d}\left(t_{L}, t_{R}\right)$ vote for the same party and agents with types $s>s_{d}\left(t_{L}, t_{R}\right)$ vote for the other party.

Proof. Without loss of generality let $t_{L}<t_{R}$. By Assumption 3 and strict concavity of $v$ in $t$ there exists at least one voter who prefers $t_{L}$ rather than $t_{R}$ (type $s_{m}$ for example) and there also exists at least a voter who prefers $t_{R}$ better than $t_{L}$ (type $s_{M}$ for example). Then, by Assumption 1, there must exist $s^{*} \in S$ such that $v\left(t_{L} ; s^{*}\right)=v\left(t_{R} ; s^{*}\right)$ and by Assumption 2 this $s^{*}$ is unique. We can show now that $s^{*}$ is the type $s_{d}\left(t_{L}, t_{R}\right)$ in the lemma. Suppose there exist $s^{\prime}<s^{*}$ and $s^{\prime \prime}<s^{*}, s^{\prime}<\mathrm{s}^{\prime \prime}$, such that $v\left(t_{L} ; s^{\prime}\right)>v\left(t_{R} ; s^{\prime}\right)$ and $v\left(t_{L} ; s^{\prime \prime}\right)<v\left(t_{R} ; s^{\prime \prime}\right)$. In this case, there must exist, by Assumption 1, $s^{\prime \prime \prime} \in S, s^{\prime}<s^{\prime \prime \prime}<s^{\prime \prime}$ such that $v\left(t_{L} ; s^{\prime \prime \prime}\right)=v\left(t_{R} ; s^{*}\right)$. But this contradicts Assumption 2 . Hence all types to the left of $s^{*}$ vote for the same party. The rest of the proof follows by a similar argument. Q.E.D.

The existence of this dividing type, $s_{d}$, will make the strategic problem of the parties simpler. The lemma also implies that, for all $t_{L} \neq t_{R}$, and given

\footnotetext{
${ }^{2}$ A possible justification for it is that there is a continuum of agents. In this case a single vote cannot affect the implemented policy. This, however, opens the question why an agent wants to vote at all. See the last section for further comments.
} 
that $E\left(t_{L}, t_{R}\right)=s_{d}$, the proportion of voters in favor of party $L$ is $\Psi\left(t_{L}, t_{R}\right)=F\left(\Omega\left(t_{L}, t_{R}\right)\right)$ and it can also be shown that whenever $t_{L}<t_{R}$ we have $\Omega\left(t_{L}, t_{R}\right)=\left[t_{m}, s_{d}\left(t_{L}, t_{R}\right)\right)$. By strict concavity of $v()$ in $t$ it must be true that $s_{d}\left(t_{L}, t_{R}\right)$ is a strictly increasing function in $t_{L}$ (also in $t_{R}$ ) whenever $t_{L} \neq t_{R}$. Therefore $s_{d}\left(t_{L}, t_{R}\right)$ is differentiable with respect to $t_{L}$ (and $t_{R}$ ) almost everywhere. So the next assumption is a weak one.

Assumption 4. $s_{d}\left(t_{L}, t_{R}\right)$ is differentiable with respect to $t_{L}$ and with respect to $t_{R}$.

Our assumptions on $s_{d}$ and $F$ imply that $\Psi\left(t_{L}, t_{R}\right)$ is strictly increasing and differentiable in $t_{L}$ and $t_{R}$ whenever $t_{L}<t_{R}$.

\section{Existence of equilibrium}

Our task now is to provide sufficient conditions for the existence of a Political Equilibrium. Let $B L\left(t_{R}\right)$ be the set of best replies of party $L$ to announcement $t_{R}$ by party $R$, i.e.,

$$
B L\left(t_{R}\right) \equiv \underset{t \in T}{\arg \max } \Pi_{L}\left(t\left(t, t_{R}\right)\right),
$$

where $t\left(t, t_{R}\right)=\Psi\left(t, t_{R}\right) t+\left(1-\Psi\left(t, t_{R}\right)\right) t_{R}$. The best reply correspondence of party $R, B R\left(t_{L}\right)$, is defined in a similar way.

To simplify the notation we will write $F\left(s_{d}\left(t_{L}, t_{R}\right)\right)$ instead of $\Psi\left(t_{L}, t_{R}\right)$ where $F$ now stands for the cumulative distribution function. The density function will be denoted by $f$. To make the proofs simpler we will also assume that $f$ is differentiable.

To study the existence of a Political Equilibrium we first show that the best reply correspondence is well defined and, second, we provide conditions to guarantee that it is single valued.

Given that $T$ is an interval, a sufficient condition for the correspondence $B L\left(t_{R}\right)$ to be well defined is that $\Pi_{L}\left(t\left(t_{L}, t_{R}\right)\right)$ is continuous in $t_{L}$. The function $t\left(t_{L}, t_{R}\right)$ is continuous except possibly at $t_{L}=t_{R}$. We now show that the function $\Pi_{L}\left(t\left(t_{L}, t_{R}\right)\right)$ is continuous in $t_{L}$. To do this it is enough to prove that it is continuous at $t_{L}=t_{R}$ :

$$
\lim _{t \rightarrow t_{R}} v\left(F\left(s_{d}\left(t, t_{R}\right)\right)\right) t_{R}+\left(1-F\left(s_{d}\left(t, t_{R}\right)\right) t_{R} ; s_{L}\right)=v\left(k t_{R}+(1-k) t_{R} ; s_{L}\right),
$$

where $k \in(0,1)$,

and this limit is equal to $v\left(F\left(s_{d}\left(t_{R}, t_{R}\right)\right)\right) t_{R}+\left(1-F\left(s_{d}\left(t_{R}, t_{R}\right)\right) t_{R}\right.$; $\left.s_{L}\right)=v\left(t_{R} ; s_{L}\right)$. Therefore $B L\left(t_{R}\right)$ is a well defined correspondence.

To simplify notation write $g\left(t_{L}, t_{R}\right)=F\left(s_{d}\left(t_{L}, t_{R}\right)\right), \quad g^{*}\left(t_{L}, t_{R}\right) \equiv$ $1-F\left(s_{d}\left(t_{L}, t_{R}\right)\right), \quad g_{1}\left(t_{L}, t_{R}\right) \equiv \delta g\left(t_{L}, t_{R}\right) / \delta t_{L}, \quad g_{2}\left(t_{L}, t_{R}\right) \equiv \delta g\left(t_{L}, t_{R}\right) / \delta t_{R} . \quad$ To guarantee that $B L\left(t_{R}\right)$ is always single valued we need some additional assumptions.

Assumption 5. The ratio $g\left(t_{L}, t_{R}\right) / g_{1}\left(t_{L}, t_{R}\right)$ is non-decreasing in $t_{L}$ and the ratio $g^{*}\left(t_{L}, t_{R}\right) / g_{2}\left(t_{L}, t_{R}\right)$ is non-increasing in $t_{R}$.

This assumption can be seen as imposing a monotone hazard rate on the functions $g\left(t_{L}, t_{R}\right)$ and $g^{*}\left(t_{L}, t_{R}\right)$. The interpretation is that as party $L$ moves its proposal $t_{L}$ toward $t_{R}$ the percentage of voters in favor of $L$ increases at a decreasing rate. 
Lemma 2. Under assumptions 1, 2, 3, 4 and 5, $B L\left(t_{R}\right)$ is single valued.

Proof. Suppose not. Then for some $t_{R}$ there exists $t^{\prime}, t^{\prime \prime} \in B L\left(t_{R}\right)$. Let $t^{\prime}<t^{\prime \prime}$. Two cases must be considered:

(i) $F\left(s_{d}\left(t^{\prime}, t_{R}\right)\right) t^{\prime}+\left(1-F\left(s_{d}\left(t^{\prime}, t_{R}\right)\right) t_{R} \neq F\left(s_{d}\left(t^{\prime \prime}, t_{R}\right)\right) t^{\prime \prime}+\left(1-F\left(s_{d}\left(t^{\prime \prime}, t_{R}\right)\right) t_{R}\right.\right.$. Suppose first that $t_{R}>l \equiv$ the ideal policy of $L$. Observe that by strict concavity of $v\left(t ; s_{L}\right)$ it must be the case that $t\left(t^{\prime}, t_{R}\right)<l<t\left(t^{\prime \prime}, t_{R}\right)$ and $t^{\prime}<l \leq t^{\prime \prime}$. We also know that given that $f$ is always greater than zero it must be the case that $t\left(l, t_{R}\right)>l$. Now $t\left(t, t_{R}\right)$ is continuous in $t$ for $t<t_{R}$. So it must exist $t^{\prime \prime \prime}, t^{\prime}<t^{\prime \prime \prime}<l$, such that $t\left(t^{\prime \prime \prime}, t_{R}\right)=l$, and this proves that $t^{\prime} \notin B L\left(t_{R}\right)$. A similar argument works for the case $t_{R}<l$. For $t_{R}=l$ it is obvious that $l=B L\left(t_{R}\right)$. It has been proven that if there is more than one element in $B L\left(t_{R}\right)$ all of them must induce the same implemented policy.

(ii) $F\left(s_{d}\left(t^{\prime}, t_{R}\right)\right) t^{\prime}+\left(1-F\left(s_{d}\left(t^{\prime}, t_{R}\right)\right) t_{R}=F\left(s_{d}\left(t^{\prime \prime}, t_{R}\right)\right) t^{\prime \prime}+\left(1-F\left(s_{d}\left(t^{\prime \prime}, t_{R}\right)\right)\right) t_{R}\right.$.

Consider first the case $l<t_{R}$. Given that $F\left(s_{d}\left(t_{L}, t_{R}\right)\right)$ is strictly monotone in $t_{L}$ and $v\left(\right.$ ) is strictly concave we have that both $t^{\prime}$ and $t^{\prime \prime}$ must be to the left of $t_{R}$. By the same argument given in (i) we know that $l \leq t^{\prime}$. Therefore we have $l \leq t^{\prime}<t^{\prime \prime}<t_{R}$ and both $t^{\prime}$ and $t^{\prime \prime}$ are in the interior of $T$; this fact and the differentiability of $v\left(F\left(s_{d}\left(t, t_{R}\right)\right) t+\left(1-F\left(s_{d}\left(t_{L}, t_{R}\right)\right) t_{R} ; s_{L}\right)\right.$ with respect to $t_{L}$ allow us to use the following first order conditions from $L$ 's maximization problem,

$$
f\left(s_{d}\left(t, t_{R}\right)\right)\left[\delta s_{d}\left(t, t_{R}\right) / \delta t\right] t+F\left(s_{d}\left(t, t_{R}\right)\right)-f\left(s_{d}\left(t, t_{R}\right)\right)\left[\delta s_{d}\left(t, t_{R}\right) / \delta t\right] t_{R}=0 .
$$

Let $\left[\delta\left(s_{d}\left(t, t_{R}\right)\right) / \delta t\right] \equiv s_{d}^{\prime}\left(t, t_{R}\right)$. Then the first order conditions can be rewritten as follows:

$$
t_{R}-t=F\left(s_{d}\left(t, t_{R}\right)\right) /\left[f\left(s_{d}\left(t, t_{R}\right)\right) s_{d}^{\prime}\left(t, t_{R}\right)\right] .
$$

Since $t^{\prime}<t^{\prime \prime}$ we have

$$
\begin{aligned}
& F\left(s_{d}\left(t^{\prime}, t_{R}\right)\right) /\left[f\left(s_{d}\left(t^{\prime}, t_{R}\right)\right) s_{d}^{\prime}\left(t^{\prime}, t_{R}\right)\right]=t_{R}-t^{\prime}>t_{R}-t^{\prime \prime} \\
& \quad=F\left(s_{d}\left(t^{\prime \prime}, t_{R}\right)\right) /\left[f\left(s_{d}\left(t^{\prime \prime}, t_{R}\right)\right) s_{d}^{\prime}\left(t^{\prime \prime}, t_{R}\right)\right]
\end{aligned}
$$

and these inequalities can be written as

$$
g\left(t^{\prime}, t_{R}\right) / g_{1}\left(t^{\prime}, t_{R}\right)=t_{R}-t^{\prime}>t_{R}-t^{\prime \prime}=g\left(t^{\prime \prime}, t_{R}\right) / g_{1}\left(t^{\prime \prime}, t_{R}\right)
$$

which is in contradiction with Assumption 5.

The case $l>t_{R}$ can be proven in a similar way. Q.E.D.

We can proceed in the same way to show that $B R\left(t_{L}\right)$ is also single valued.

In the following, we show that under the assumptions introduced so far a Political Equilibrium always exists. The second part of Theorem 3 shows that in equilibrium parties propose different policies. The last part states that whenever the equilibrium is an interior one, both parties obtain the same number of votes ${ }^{3}$. This implies that the "median" voter is indifferent between

\footnotetext{
${ }^{3}$ This last result depends very much on the proportional representation assumption. In a more general model with weights different from $W_{P R}$ this wouldn't be true.
} 
the two equilibrium policies. It can be easily shown, however, that in general $t\left(t_{L}^{*}, t_{R}^{*}\right)$ will be different from $t\left(s_{M V}\right)$. Thus not only the equilibrium strategies of parties differ from the ones in the standard model but the outcome of the game as well.

\section{Theorem 3. Suppose Assumptions 1-5 hold. Then}

(i) a Political Equilibrium, $\left(t_{L}^{*}, t_{R}^{*}\right)$, exists,

(ii) $t_{L}^{*}<t_{R}^{*}$

(iii) If $t_{m}<t_{L}^{*}<t_{R}^{*}<t_{M}$ then $s_{d}\left(t_{L}^{*}, t_{R}^{*}\right)=s_{M V}$.

Proof. (i) Let $B: T \times T \rightarrow T \times T$ be defined by $B\left(t_{L}, t_{R}\right)=\left(B L\left(t_{R}\right), B R\left(t_{L}\right)\right)$. By Lemma 2, B is single valued. To apply Brower's Fixed Point Theorem we only need to show that $B$ is continuous. Continuity of $B$ follows from the continuity of $\Pi_{L}\left(t\left(t_{L}, t_{R}\right)\right)$ and $\Pi_{R}\left(t\left(t_{L}, t_{R}\right)\right)$ in $\left(t_{L}, t_{R}\right)$ and Berge's Maximum Theorem. (ii) Suppose they were proposing the same policy, say $t^{\prime}$, and that w.l.o.g. $1<t^{\prime}$. Clearly $t\left(t^{\prime}, t^{\prime}\right)=t^{\prime}>t\left(l, t^{\prime}\right)>l$. Thus party $L$ would be better off proposing $l$ rather than $t^{\prime}$ and hence $\left(t^{\prime}, t^{\prime}\right)$ cannot be an equilibrium. It is not difficult to see that $t_{R}<t_{L}$ cannot be an equilibrium either. (iii) When the equilibrium policies belong to the interior of $T$ the first order conditions of the maximization problems imply that $F\left(s_{d}\left(t_{L}^{*}, t_{R}^{*}\right)\right)=1-\left(F\left(s_{d}\left(t_{L}^{*}, t_{R}^{*}\right)\right)\right)$, hence $F\left(s_{d}\left(t_{L}^{*}, t_{R}^{*}\right)\right)=0.5$. Q.E.D.

It is important to realize that our existence proof depends very much on Assumption 5. Unfortunately, it is questionable whether this is a natural assumption. The problem with this assumption is that imposes a joint restriction on the set of admissible utility functions and the set of admissible distributions of types. Note that the very standard condition of increasing hazard rate of the distribution function of $s$ is not enough for Assumption 5 to hold. It is also needed that $s_{d}^{\prime}$ changes in the right direction.

\section{Symmetric preferences}

Many models analyzed in the literature consider the case of symmetric preferences. An agent with symmetric preferences always votes for the closest proposal to his ideal policy (see for example Eaton and Lipsey (1975) and Denzau (1985) where agents have symmetric preferences and the distribution of ideal points is uniform; see also Cox (1987) where this distribution is not necessarily uniform). Through this section we will assume that agents have symmetric preferences. The reason why we study this more restrictive model is twofold. First, if we assume this type of preferences, monotonicity on the hazard rate of the distribution function of ideal policies is sufficient to guarantee the existence of equilibrium. Second, we will be able to compare our results with the ones obtained under other voting models.

Assumption 6 (Symmetric preferences). For all $s \in S$ and for all $x$ such that $t(s)+x \in T, t(s)-x \in T$, we have $v(t(s)+x ; s)=v(t(s)-x ; s)$.

Assumption 7. Voter ideal policies, $t(s) \equiv q$, are distributed according to a continuous distribution function $F(q)$ with differentiable density function $f(q)$. Furthermore, the ratio $F(q) / f(q)$ is non-decreasing in $q$ and the ratio $(1-F(q)) / f(q)$ is non-increasing in $q$. 
This assumption is equivalent to requiring that both $\log F$ and $\log (1-F)$ be concave. This condition is weaker than concavity of $\log f$ (see Barlow and Proschan 1975). Most commonly used density functions are log-concave and therefore satisfy Assumption 7 (see Caplin and Nalebuff 1991 for a list of the class of log-concave densities and Pratt 1981 for examples of distribution functions for which $\log (1-F)$ and $\log F$ are concave but $\log f$ is not).

Theorem 4. Under Assumptions 1, 2, 3, 4, 6 and 7

(i) a Political Equilibrium, $\left(t_{L}^{*}, t_{R}^{*}\right)$, exists,

(ii) $t_{L}^{*}<t_{R}^{*}$, and

(iii) if $t_{m}<t_{L}^{*}<t_{R}^{*}<t_{M}$ then $s_{d}\left(t_{L}^{*}, t_{R}^{*}\right)=s_{M V}$ and $t\left(t_{L}^{*}, t_{R}^{*}\right)=t\left(s_{M V}\right)$.

Proof. By Assumption 6, the ideal policy of the "dividing" type is $t\left(s_{d}\left(t_{L}, t_{R}\right)\right)=\left(t_{R}+t_{L}\right) / 2$. All the agents with ideal policy to the left of $\left(t_{R}+t_{L}\right) / 2$ will vote for $L$. In this case we have that

$$
F\left(t\left(s_{d}\left(t_{L}, t_{R}\right)\right)\right) /\left[f\left(t\left(s_{d}\left(t_{L}, t_{R}\right)\right)\right) s_{d}^{\prime}\left(t_{L}, t_{R}\right)\right]=2 F\left(0.5\left(t_{L}+t_{R}\right)\right) /\left[f\left(0.5\left(t_{L}+t_{R}\right)\right)\right] .
$$

Assumption 7 implies that this ratio is non-decreasing in $t_{L}$. It is not difficult to see that such ratio is the same one we used in Assumption 5, $g\left(t_{L}, t_{R}\right) / g_{1}\left(t_{L}, t_{R}\right)$ (in the same way we can obtain a ratio similar to $g^{*}\left(t_{L}, t_{R}\right) / g_{2}\left(t_{L}, t_{R}\right)$ so that all the conditions of Theorem 3 are also satisfied here). Thus, only the last equality in (iii) remains to be proven. At equilibrium both parties obtain the same number of votes. Hence the dividing type must be the median voter and, by symmetry of preferences, the implemented policy coincides with the median voter's ideal policy. Q.E.D.

It is important to remark that here the equilibrium implements the policy most preferred by the median voter. This fact depends very much on the symmetry of the preferences and, as noted in the previous section, is not true for more general models. The equilibrium policies, however, do not converge.

The non-convergence result is also obtained in other models considering ideological parties (Wittman 1983, 1990; Calvert 1985; Roemer 1993, 1994; Shultz 1995). All these models assume that there is uncertainty about the distribution of voter preferences (consequently the outcome of an election is probabilistic) and that the implemented policy is the one proposed by the winner. We will show that our approach not only provides a different political equilibrium from the one associated to these probabilistic models but what is even more important, it captures a different kind of trade-off parties might face when choosing their proposals.

In the probabilistic case, given the proposals $\left(t_{L}, t_{R}\right)$ party $L$ wins the election with probability $p\left(t_{L}, t_{R}\right)$. This probability can be defined in several different ways and is always assumed to be decreasing in the distance between $t_{L}$ and $t_{R}$. One possibility is to make $p\left(t_{L}, t_{R}\right)=F\left(t\left(s_{d}\left(t_{L}, t_{R}\right)\right)\right)$ so that the weights in our model are now the probabilities of winning the election. Regardless of the specific function used to defined $p\left(t_{L}, t_{R}\right)$ we have that on the one hand party $L$ has an incentive to choose a policy that is close to his ideal one. On the other hand, a policy too close to the ideal one may induce a very low probability of winning the election and since the implemented policy is the one proposed by the winner $L$ also has an incentive to move toward the policy announced by $R$. As a result of these two forces we have that in all these 
probabilistic models: (i) at the equilibrium parties do not converge in their policies, (ii) the equilibrium policies lie in between the ideal policies $l$ and $r$.

To see the result in (ii) suppose that party $L$ is proposing a policy $t_{L}<l$. It is clear that $t_{L}$ cannot be part of an equilibrium: moving $t_{L}$ toward $t_{R}$ increases $p\left(t_{L}, t_{R}\right)$, the probability of winning, and if $L$ wins the implemented policy is closer to its ideal policy $l$. Hence that movement toward $t_{R}$ increases the expected utility of party $L$.

These probabilistic models and our Proportional Representation model only share point (i) above in common but not (ii). This discrepancy is due to the fact that in our model the proposal of the defeated party always has some direct influence on the implemented policy. The following specific example will help to see the importance of this difference.

Suppose that $t_{m}=0, t_{M}=1$. Let $f$, the density of ideal policies, be triangular $^{4}$ and symmetric, i.e., $f(x)=4 x$ for $0 \leq x \leq 1 / 2$ and $f(x)=4(1-x)$ for $1 / 2 \leq x \leq 1$. In this case, the median and the mean ideal policies are both equal to $1 / 2$. Let parties' ideal policies be such that $1 / 4<l<1 / 2<r<3 / 4$. Under any of the equilibrium probabilistic models mentioned above the equilibrium policies, $\left(t_{L}^{\prime}, t_{R}^{\prime}\right)$, have to lie in between $l$ and $r$, i.e., $l<t_{L}^{\prime}<t_{R}^{\prime}<r$. In our model, on the contrary, straightforward calculations show that the unique Political Equilibrium is $\left(t_{L}^{*}=1 / 4, t_{R}^{*}=3 / 4\right)$. Thus, parties propose platforms that are more radical than their ideal policies. This happens even for the cases in which a majority of voters lie in between $l$ and $r$.

More dramatic examples are also possible. For instance, if the density function $f$ is either uniform or $\mathrm{U}$-shaped it can be shown that the unique Political Equilibrium in our model is $\left(t_{L}^{*}=0, t_{R}^{*}=1\right)$. Thus, when there are more voters close to the extremes of the political spectrum than in the middle, the Political Equilibrium is a complete radical one (but not the implemented policy that continues to be in the middle.) Under the probabilistic models, however, the equilibrium policies remain in the center.

\section{Final comments}

We have analyzed a very simple theoretical model of party competition with two main distinctive features: (i) parties are ideological, (ii) the policy implemented after the election does not need to coincide with the one proposed by the winner. We take the view that in many democratic societies minorities also have some influence on the policies implemented. Thus the policy adopted should be a "compromise" that considers all the proposals made by the different parties. The weight given to each proposal is related to the popular support obtained by each party. In particular we have analyzed the proportional representation case in which the weights are proportional to the number of votes.

It has been shown that parties have incentives to diverge in their proposals. Sometimes these incentives may be strong enough to make parties to

\footnotetext{
${ }^{4}$ The fact that $f$ is not differentiable at $x=1 / 2$ is not relevant for the present example.
} 
adopt very radical policies. This result makes our approach different from other models in which parties are also ideological.

Voters in our model behave in a non-strategic way. An agent always votes for the "closest" proposal and does not take into account the possible influence in the implemented policy. If there is a continuum of agents this seems a reasonable assumption since any single vote has a negligible effect on the implemented policy. It is also reasonable to assume that in real cases voters are less well informed than parties about the way the electoral outcome induces the specific weights used to determine the implemented policy. If instead the Proportional Representation case we consider a more realistic one in which those weights are given by nonlinear functions this assumption would be even more reasonable. The implications of allowing for more sophisticated type of voters are left for future research.

Additional future work should also consider a more realistic function $W$ and provide a better justification of the assumption that the implemented policy is a compromise between the two announced policies and not a compromise in between the two ideal policies of the parties. This compromise could be reached in the space of utilities rather than in the policy space. The analysis should also be extended to the multiparty and multidimensional policy space cases.

\section{References}

Barlow RE, Proschan F (1981) Statistical theory of reliability and life testing. To Begin With, Silver Spring, MD

Barnett W, Hinich M, Schofield NJ (1993) (eds) Political economy, institutions, competition, and representation. C.U.P., Cambridge

Calvert RL (1985) Robustness of the multidimensional voting model: candidates motivations, uncertainty, and convergence. Am J Polit Sci 29: 69-95

Caplin A, Nalebuff B (1991) Aggregation and social choice: a mean voter theorem. Econometrica 59(1): 1-23

Cox GW (1987) Electoral equilibrium under alternative voting institutions. Am J Polit Sci 31: 82-108

Denzau A, Kats A, Slutsky S (1985) Multi-agent equilibria with market share and ranking objectives. Soc Choice Welfare 2: 95-117

Downs A (1957) An economic theory of democracy. Harper, New York

Eaton BC, Lipsey R (1975) The principle of minimum differentiation reconsidered: some new developments in the theory of spatial competition. Rev Econ Studies 42: $27-50$

Enelow J, Hinich M (1990) (eds) Advances in the spatial theory of voting. C.U.P., Cambridge

Ginsburgh V, Pestieau P, Thisse J-F (1987) A spatial model of party competition with electoral and ideological objectives. In: Gosh A, Rushton G, Spatial analysis and location-allocation models. Van Nostrand Reinhold, New York

Hotelling H (1929) Stability in competition. Econ J 39: 41-57

Poole KT, Rosenthal H (1984) US Presidential Elections 1968-1980: a spatial analysis. Am J Polit Sci 28

Poole KT, Rosenthal H (1991) Patterns of congressional voting. Am J Polit Sci 35

Pratt J (1981) Concavity of the log likelihood. J Am Statist Assoc 76(373): 103-106 
Roemer JE (1993) Political-economic equilibrium when parties represent constituents: the unidimensional case. W.P. \#93-19, Department of Economics, UCDavis

Roemer JE (1994) A theory of policy differentiation in single issue electoral politics. Soc Choice Welfare 11(4): 355-380

Shultz C (1993) The politics of persuasion when voters are rational. Scandinavian J Econ 97(3): 357-368

Wittman D (1983) Candidate motivation: a synthesis of alternative theories. Am Polit Sci Rev 77: 142-157

Wittman D (1990) Spatial strategies when candidates have policy preferences. In: Enelow JM, Hinich MJ (eds) Advances in Spatial Theory of Voting. Cambridge Univeristy Press, Cambridge 